\title{
Association between polymorphism of optic disc related genes and susceptibility to primary open angle glaucoma in Inner Mongolia
}

\author{
Xiangyang Xin, Peng Chen, Chenlu Liu \\ Department of Ophthalmology, The Third Affiliated Hospital of Inner Mongolia Medical University (Baogang Hospital), \\ Baotou, Inner Mongolia, China
}

Received: September 23, 2018

Accepted: November 14, 2018

Online Published: December 10, 2018

DOI: $10.14725 /$ dcc.v5n4p30

URL: http://dx.doi.org/10.14725/dcc.v5n4p30

\begin{abstract}
Objective: To explore the relationship between the polymorphism of optic disc related genes and the susceptibility to primary open angle glaucoma (POAG) in Inner Mongolia.

Methods: A retrospective study was adopted to include 108 patients who were diagnosed as POAG in six hospitals in Hohhot and Baotou from January of 2014 to December of 2016 (POAG group). At the same time, 120 healthy examinees were included in the control group. 1-2 $\mathrm{ml}$ of whole blood was collected by use of EDTA anticoagulation tubes from each patient in these two groups. It was required to fully mix EDTA with whole blood in order to extract genomic DNA and place it in the $-20^{\circ} \mathrm{C}$ refrigerator. Mass spectrometry was used to identify the genotype of single nucleotide polymorphism (SNP) of RFTN1 (rs690037), ATOH7 (rs7916697, rs3858145), CDC7 (rs1192415), CDKN2B (rs1063192) and SIX (rs10483727) in 108 POAG patients and 120 normal controls. $\chi^{2}$ test and binary logistic regression were used to analyze the relationship between the genetic polymorphism and the occurrence of POAG.

Results: The frequency of G allele at CDKN2B (rs1063192) in POAG group was significantly higher than that in the control group $(27 \%$ vs. $17 \%)$, and the difference was of statistical significance [odds ratio $(\mathrm{OR})=1.824,95 \%$ confidence interval (CI): $1.163-2.861, p=.008]$; As to the comparison in the frequency of allele at the other 7 SNPs between the two groups, the differences were statistically significant (all $p>.05$ ). Additive and dominant models at rs 1063192 indicated that the individuals with G allele were more susceptible to POAG, and the difference was of statistical significance $(p<.05)$, and recessive models showed that the risk in the individuals with A allele was not significantly reduced, and the difference was of no statistical significance $(p>.05)$. There was no statistically significant difference in the distribution of genotypes at other SNPs between POAG group and the control group $(p>.05)$.

Conclusions: The genetic polymorphism of CDKN2B (rs1063192) is associated with the susceptibility to POAG, and G allele may increase the risk for POAG.
\end{abstract}

Key Words: Primary open angle glaucoma, Optic disc area, Vertical cup disc ratio, Polymorphism

Glaucoma is a multifactorial disease caused by a variety of environmental factors and genes. ${ }^{[1]}$ Primary open angle glaucoma (POAG) accounts for more than 50\% in all types of glaucoma, ranking the second place in the table of blinding diseases, and has the characteristics of heredity, progressivity and irreversibility. ${ }^{[2]}$ The manifestation of POAG is presented as elevated intraocular pressure with anterior chamber angle always open. The progressive stage

*Correspondence: Xiangyang Xin; E-mail: xinxiangyang_2006@163.com; Address: Department of Ophthalmology, The Third Affiliated Hospital of Inner Mongolia Medical University (Baogang Hospital), Baotou, Inner Mongolia, China. 
is characterized by progressive atrophy of optic disc cupping, painless vision loss and glaucomatous visual field defect, which may eventually lead to blindness. Due to the lack of typical clinical symptoms, glaucoma in the early stage may be misdiagnosed as fatigue, migraine and gastroenteritis, so that it is not treated in time or ignored (only one eye is given treatment). Therefore, it will not be noticed until the loss of vision even visual field defect in a considerable scale gradually appear, at that time, the loss of visual function is irreversible. POAG is a main type of glaucoma occurring in African, European and American people. Nevertheless, primary angle-closure glaucoma (PACG) is a common glaucoma type in Asian people. Due to the covert onset of POAG and the lack of specificity in its early diagnosis, the patients' visual function is seriously damaged when they admit to the hospital. Even if combined medication or surgical treatment is given, it fails to control the intraocular pressure effectively, delay the optic atrophy or control the intraocular pressure within the normal range, but the optic damage remains to be progressive. Therefore, the early diagnosis and treatment of POAG can maintain the present vision at the maximum and delay the progression of glaucoma. POAG is not only related to hypertension, diabetes mellitus, family history, smoking and other risk factors, but also related to heritable and genetic variations. ${ }^{[3]}$ It is found that the changes in optic disc area and vertical cup disc ratio (VCDR) precede those in visual field and have a certain heritability. Therefore, it is of great significance to study the relationship between the polymorphism of the optic disc related genes and the susceptibility to POAG. So far, it has reported more than 20 genes or sites that are related to the pathogenesis of POAG, ${ }^{[4]}$ such as CAV1, CAV2, CDKN2B-AS1, CDKN2A, CDKN2B and TMCO1. ${ }^{[5,6]}$ Most of these sites are screened out by genome-wide association study (GWAS), and there are few reports aimed at Chinese people. Considering the differences in region and race, this research was designed to study the relationship of the optical disc area and the polymorphism of VCDR related genes [RFTN1 (rs690037), ATOH7 (rs7916697, rs3858145), CDC7 (rs1192415), CDKN2B (rs1063192) and SIX (rs10483727)] with the susceptibility to POAG in Chinese population.

\section{Data and methods}

\subsection{General information}

A retrospective study was adopted to include 108 cases of patients who were diagnosed as POAG in six hospitals (Baogang Honspital, the First Affiliated Hospital of Baotou Medical College, Inner Mongolia International Mogolian Hospital, Baotou Eye Hospital, Baotou Chaoju Ophthalmology Hospital and Hohhot Chaoju Ophthalmology Hospital) in Hohhot and Baotou in Inner Mongolia Autonomous Region from January of 2014 to December of 2016. There were

Published by New Century Science Press
60 cases of male patients and 48 cases of female patients at the age of $(49.67 \pm 8.26)$ with a course of $(3.2 \pm 1.5)$ years. Inclusion criteria for patients in POAG group: (1) Under high intraocular pressure, it was observed that the chamber angle was in an open state with the help of gonioscopy; (2) The intraocular pressure exceeded $21 \mathrm{mmHg}(1 \mathrm{mmHg}=$ $0.133 \mathrm{kPa}$ ) two times in $1 \mathrm{~d}$ at least, and the fluctuation of intraocular pressure was more than $6 \mathrm{mmHg}$ within 1 day; (3) Fundus examination: $\mathrm{C} / \mathrm{D} \geq 0.3$, with optic disc changes to different degrees; (4) Visual field examination revealed different degrees of glaucomatous visual field defect; (5) OCT results revealed the damage in the retinal nerve fiber layer.

In the same period, 120 healthy subjects were included in the control group, which consisted of 54 male patients and 66 female patients at the age of $(47.41 \pm 14.32)$. Inclusion criteria for patients in the control group: (1) The intraocular pressure was less than or equal to $21 \mathrm{mmHg}$; (2) Peripheral anterior chamber depth was more than $1 / 2$ corneal thickness (CT) and axial anterior chamber depth was more than $2 \mathrm{CT}$; (3) Fundus examination: C/D was less than or equal to 0.3 ; (4) Microscopy and ophthalmoscopy showed no refractive media opacity, no other eye diseases (except for ametropia) and family history of glaucoma; (5) Normal pupil size; (6) Normal chromatic vision; (7) B-scan ultrasonography showed no intraocular lesions such as retinal detachment or lens dislocotion; (8) OCT examination revealed no defect in the retinal nerve fiber layer or fundus lesions. According to the principle of frequency matching, there were no statistically significant differences in gender and age composition between POAG group and the control group (all $p>.05$ ). The research was approved by Ethics Committee of Baogang Hospital and all patients signed informed consent forms.

\subsection{Methods}

1-2 $\mathrm{ml}$ fasting venous blood was taken from the patient's elbow in each patient in these two groups with EDTA anticoagulation tubes, overturned and blended for 8-10 times to make sure EDTA and whole blood were fully mixed up for the optimal anticoagulant effect, and placed in a $-80^{\circ} \mathrm{C}$ refrigerator for cryopreservation. Whole blood DNA purification kits (Beijing Cwbiotech Co., Ltd.) were used to extract genomic DNA, which was placed in a $-20^{\circ} \mathrm{C}$ refrigerator. PCR reagent kits (Agena Bioscience, USA) were applied to PCR. Assay Design Suite v2.0, an online primer designing software (Agena Bioscience, USA) was used to automatically design PCR primers and extension primers for genotyping single nucleotide polymorphism (SNPs). MALDI mass spectrometer was applied to the analysis of samples to be tested. MassARRAY Typer software automatically interprets the peak of relative molecular mass detected by mass spectrometry and converts it into mass spectrum corresponding to SNP sites for genotyping. 


\subsection{Statistical methods}

SPSS 17.0 statistical software was applied to statistical analysis. Chi-square test or Fisher's exact test was used in the analysis of the difference in allele frequency of each SNP site in POAG group and the control group. Binary logistic regression was applied to the correlation analysis of genotype and disease, with age and gender adjusted to calculate $p$ value, odds ratio (OR) and $95 \%$ confidence interval $(95 \%$ $\mathrm{CI})$. The regression analysis was carried out according to different genetic models. It was assumed that there were three genotypes: AA, Aa and aa, if "a" was considered as a risk gene, additive model meant as long as there was one "a" in the genotype, the frequency was counted. Therefore, the frequencies of AA, Aa and aa were 0,1 and 2. Dominant model required that the frequency was counted as long as there was one "a" in the genotype. The corresponding frequencies of AA, Aa and aa were 0,1 and 1 respectively. In recessive model, if two "a" were in the genotype, the frequency was counted. Therefore, the frequencies of AA, Aa and aa were 0,0 and 1 respectively. The difference $(p<.05)$ was of statistical significance.

\section{Results}

\subsection{Allele frequency distribution of SNP sites in the optic disc related genes}

The genotype distribution of SNPs in the optic disc related genes in the control group was consistent with HardyWeinberg equilibrium (HWE) $(p>.05)$, indicating that the research data were of group representativeness. $\chi^{2}$ test was applied to the analysis of allele frequency distribution of SNP sites in the optic disc related genes in POAG group and the control group, and the results confirmed that the frequency of $G$ allele at CDKN2B (rs1063192) in POAG group was obviously higher than that in the control group $(\mathrm{OR}=1.824$, 95\% CI: 1.163 $2.861, p=.008)$. However, as to the frequency of allele at RFTN1 (rs690037), ATOH7 (rs7916697, rs3858145), CDC7 (rs1192415) and SIX (rs10483727), the differences between these two groups were not statistically significant (all $p>.05)$ (see Table 1).

\subsection{The relationship between genotype distribution of SNP and POAG}

Binary logistic regression was used to analyze the genotype distribution of SNP in the optic disc related genes in POAG group and the control group to figure out dominant, recessive and additive models, with age and gender adjusted. The additive model at CDKN2B (rs1063192) site showed that the individuals with G/A genotype were 1.81 times and the individuals with G/G genotype were 2.60 times more susceptible to POAG than the individuals with A/A genotype. The difference was statistically significant $(p<.05)$. The dominant model showed that, in comparison with the individuals with $\mathrm{A} / \mathrm{A}$, the individuals with $\mathrm{G}$ allele were 1.94 times more susceptible to POAG, and the difference was of statistical significance $(p<.05)$. However, the recessive model showed no significant decrease in the risk for POAG in the individuals with A allele in comparison with the individuals with $\mathrm{G} / \mathrm{G}(p>.05)$, indicating that $\mathrm{G}$ allele at CDKN2B (rs1063192) site could be a risk gene for POAG, but A allele was not a protective gene. Nevertheless, there was no statistically significant difference in the distribution of other SNP genotypes between POAG group and the control group $(p>.05)$, indicating that alleles at ATOH7 (rs7916697), ATOH7 (rs3858145), CDC7 (rs1192415), SIX (rs10483727) and RFTN1 (rs690037) sites were neither POAG risk genes nor protective genes (see Table 2).

Table 1: Allele frequency of SNP in optic disc related genes in two groups

\begin{tabular}{|c|c|c|c|c|c|c|}
\hline \multirow{2}{*}{ Gene } & \multirow{2}{*}{ SNPs } & \multirow{2}{*}{ Allele (M/m) } & \multicolumn{2}{|c|}{ Allele Frequency } & \multirow{2}{*}{ OR $(95 \% \mathrm{CI})$} & \multirow{2}{*}{$p$ value } \\
\hline & & & POAG Group & Control Group & & \\
\hline \multirow[t]{2}{*}{ CDKN2B } & \multirow[t]{2}{*}{ rs1063192 } & $\mathrm{A}$ & $157(73 \%)$ & $199(83 \%)$ & 1.824 & \multirow{2}{*}{.008} \\
\hline & & $\mathrm{G}$ & $59(27 \%)$ & $41(17 \%)$ & $(1.163-2.861)$ & \\
\hline \multirow[t]{2}{*}{ ATOH7 } & \multirow[t]{2}{*}{ rs3858145 } & A & $142(66 \%)$ & $168(70 \%)$ & 1.216 & \multirow{2}{*}{.330} \\
\hline & & $\mathrm{G}$ & $74(34 \%)$ & $72(30 \%)$ & $(0.820-1.803)$ & \\
\hline \multirow[t]{2}{*}{ ATOH7 } & \multirow[t]{2}{*}{ rs7916697 } & $\mathrm{G}$ & $150(69 \%)$ & $182(76 \%)$ & 1.381 & \multirow{2}{*}{.126} \\
\hline & & A & $66(31 \%)$ & $58(24 \%)$ & $(0.913-2.088)$ & \\
\hline \multirow[t]{2}{*}{$\mathrm{CDC} 7$} & \multirow[t]{2}{*}{ rs1192415 } & A & $168(78 \%)$ & $200(83 \%)$ & 1.429 & \multirow{2}{*}{.133} \\
\hline & & $\mathrm{G}$ & $48(22 \%)$ & $40(17 \%)$ & $(0.895-2.279)$ & \\
\hline \multirow[t]{2}{*}{ SIX } & \multirow[t]{2}{*}{ rs10483727 } & $\mathrm{C}$ & $168(78 \%)$ & $171(71 \%)$ & 0.708 & \multirow{2}{*}{.111} \\
\hline & & $\mathrm{T}$ & $48(22 \%)$ & $69(29 \%)$ & $(0.463-1.084)$ & \\
\hline \multirow[t]{2}{*}{ RFTN1 } & \multirow[t]{2}{*}{ rs690037 } & $\mathrm{C}$ & $93(43)$ & $123(51 \%)$ & 1.390 & \multirow{2}{*}{.080} \\
\hline & & $\mathrm{T}$ & $123(57 \%)$ & $117(49 \%)$ & $(0.961-2.012)$ & \\
\hline
\end{tabular}

Note. SNPs: single nucleotide polymorphism; POAG: primary open angle glaucoma; OR: odds ratio; CI: confidence interval 
Table 2: Genotype distribution in SNP sites in optic disc related genes in two groups

\begin{tabular}{|c|c|c|c|c|c|c|c|}
\hline \multirow{2}{*}{ Gene } & \multirow{2}{*}{ SNPs } & \multicolumn{2}{|c|}{ Additive Model } & \multicolumn{2}{|c|}{ Dominant Model } & \multicolumn{2}{|c|}{ Recessive Model } \\
\hline & & $p$ value & OR $(95 \%$ CI) & $p$ value & OR $(95 \%$ CI) & $p$ value & OR $(95 \% \mathrm{CI})$ \\
\hline CDKN2B & rs 1063192 & .047 & $2.60(0.91-7.41)$ & .017 & $1.94(1.13-3.36)$ & .137 & $2.16(0.77-3.25)$ \\
\hline ATOH7 & rs3858145 & .594 & $0.77(0.34-1.78)$ & .276 & $1.34(0.79-2.25)$ & .766 & $0.89(0.40-1.96)$ \\
\hline ATOH7 & rs7916697 & .132 & $1.30(0.79-2.14)$ & .124 & $1.51(0.90-2.55)$ & .469 & $1.43(0.54-3.79)$ \\
\hline $\mathrm{CDC} 7$ & rs1192415 & .104 & $4.82(0.99-23.48)$ & .393 & $1.27(0.73-2.20)$ & .074 & $4.72(0.98-22.74)$ \\
\hline SIX & rs10483727 & .235 & $0.64(0.23-1.78)$ & .089 & $0.63(0.37-1.07)$ & .595 & $0.76(0.28-2.08)$ \\
\hline RFTN1 & rs690037 & .258 & $1.75(0.88-3.47)$ & .243 & $1.42(0.79-2.58)$ & .123 & $1.55(0.89-2.71)$ \\
\hline
\end{tabular}

Note. SNPs: single nucleotide polymorphism; OR: odds ratio; CI: confidence interval

\section{Discussion}

The pathogenesis of POAG is not completely clarified and it is generally believed to be the result of the interaction between multiple genes and the environment. POAG is characterized by the changes in the structure and the function of optic disc, ultimately leading to optic nerve atrophy. Therefore, the early diagnosis and prevention of POAG is of great significance.

The present study confirms that the changes in the structure and the function of optic disc precede those in visual field Hence, the measurement of optical disc parameters, including optic disc area and VCDR, can contribute to the early diagnosis of POAG. ${ }^{[7]}$ The enlargement of optical disc area is a risk factor for the onset of POAG. ${ }^{[8]}$ Abnormal enlargement of VCDR is also an important factor in the pathogenesis of POAG.

The heritability of optic disc area and VCDR can reach up to $52 \%-59 \%$ and $48 \%-80 \%$ respectively, indicating that POAG has a hereditary susceptibility. ${ }^{[9]}$ Some studies have found that optic disc related genes and polymorphic sites are associated with the susceptibility to POAG in Caucasian and Asian population, and SNP sites can be used as biological markers for the early diagnosis and risk prediction of POAG. Macgregor et al. ${ }^{[10]}$ found in the research on GWAS in two groups of twins in Australia, ATOH7 (rs3858145) and RFTN1 (rs9607429) were associated with the optic disc area. Further combined with literature reports for Metaanalysis, it confirmed two sites - rs190004 and rs690037 - of ATOH7 were also associated with the optic disc area. Ramdas et al. ${ }^{[11]}$ have made a Meta-analysis of GWAS data and screened out two sites associated with the optic disc area: CDC7 (rs1192415) and ATOH7 (rs1900004); and two sites associated with VCDR: CDKN2B (rs1063192) and SIX (rs10483727). Fan et al. ${ }^{[12]}$ have analyzed related genotypes in 539 white patients with POAG and 336 normal subjects in USA, and found that the polymorphism of
CDKN2B (rs1063192) and SIX (rs10483727) are associated with POAG, and rs 1063192 is associated with small VCDR, which could reduce the risk, while rs10483727 is associated with large VCDR, which could increase the risk for the disease. Burdon et al. ${ }^{[6]}$ have made a GWAS study to European-Australian individuals and revealed another site which is considered to be associated with optic disc, it is at the antisense strand RNA1 of CDKN2B, named rs497756 (CDKN2B-AS1) and located near CDKN2B (rs1063192). It can regulate the expression of $\mathrm{CDKN} 2 \mathrm{~A}$ and $\mathrm{CDKN} 2 \mathrm{~B}$ genes. Cao et al. ${ }^{[13]}$ screened out 13 genes and 18 sites (including the above genes and sites) related to optic disc in the Afro-Caribbean, and only the polymorphism of CDKN2B (rs1063192) was found to be related to POAG.

In this study, the genotype analysis was conducted to $6 \mathrm{SNP}$ sites in 108 POAG patients in Inner Mongolia Autonomous Region (Hohhot and Baotou) and 120 normal subjects. Only the polymorphism of CDKN2B (rs1063192) was found to be significantly correlated with the susceptibility to POAG. CDKN2B, namely, cyclin dependent kinase inhibitor 2B, can regulate cell proliferation, differentiation, cell cycle and apoptosis with the help of transforming growth factor (TGF- $\beta$ ), and it is associated with some diseases such as neuroglioma, diabetes and acute myocardial infarction. ${ }^{[14-16]}$ TGF- $\beta$ is a type of multi-functional cytokines synthesized by a variety of tissue cells, secreted in many of optical tissues. It can promote the proliferation of extracellular matrix and fibroblasts, resulting in increased intraocular pressure and inducing glaucoma. ${ }^{[15]}$

rs 1063192 is located in the $3^{\prime}$ untranslated region (UTR) of CDKN2B gene, and 3' UTR has binding targets of miRNA or siRNA. Binding to the target may cause mRNA degradation or terminate translation, and regulate the protein expression of the target gene. Therefore, many experts are dedicated to the research on the polymorphism of CDKN2B (rs1063192) and CDKN2B-AS1 (rs4977757) and the susceptibility to POAG. However, G allele at CDKN2B 
(rs1063192) that increases the risk for neuroglioma, diabetes and acute myocardial infarction can significantly reduce the risk for POAG. Cao et al. ${ }^{[13]}$ studied the genotype of CDKN2B (rs1063192) in 437 Afro-Caribbean people (including 272 POAG patients) and found that $\mathrm{G}$ allele only accounted for $3.9 \%$ in POAG group, and reached up to $9.4 \%$ in the control group. The difference was statistically significant. Fan et al. ${ }^{[12]}$ also drew the same conclusion from the study to American white people, indicating that $\mathrm{G}$ allele at CDKN2B (rs1063192) was found to be a protective gene for POAG.

This research team first studied the relationship between the genotype distribution of RFTN (rs690037), ATOH7 (rs7916697, rs3858145), CDC7 (rs1192415), CDKN2B (rs1063192) and SIX (rs10483727) and the susceptibility to POAG in Asian population. The results showed that $\mathrm{G}$ allele at CDKN2B (rs1063192) significantly increased the risk for POAG in the Asian, and the analysis of recessive, dominant and additive models indicated that $\mathrm{G}$ allele was a risk gene, but A allele was not a protective gene for POAG, which seemed to be contrary to the research results acquired by Fan et al. ${ }^{[12]}$ and Cao et al. ${ }^{[13]}$ It was mainly because of different research objects. Fan et al. ${ }^{[12]}$ made a study aimed at American white people, Cao et al. ${ }^{[13]}$ took AfroCaribbean people as research objects, but the research objects were the Asian (including Mongolian people and Han people in Northern China). Gene polymorphism is closely related to factors such as nationality, ethnicity and region,

\section{References}

[1] Bron A, Chnine G, Villain M, et al. Risk factors for primary openangle glaucoma. J Fr Ophtalmol. 2008; 31(4): 435-444. https: //doi.org/10.1016/S0181-5512(08)71443-8

[2] Zhang LN. Meta-analysis on the polymorphism of rs2165241C/T at LOXL1 and the susceptibility to open angle glaucoma. Zhejiang Clinical Medical Journal. 2013; 10(10): 1553-1554.

[3] Fan BJ, Wang DY, Pasquale LR, et al. Genetic variants associated with optic nerve vertical cup-to-disc ratio are risk factors for primary open angle glaucoma in a US Caucasian population. Invest Ophthalmol Vis Sci. 2011; 52(3): 1788-1792. PMid: 21398277. https://doi.org/10.1167/iovs.10-6339

[4] Komaromy AM, Petersen-Jones SM. Genetics of primary open angle glaucoma. J Ophthalmol. 2014; 58(1): 1-15.

[5] Thorleifsson G, Waiters GB, Hewitt AW, et al. Common variants near CAV1 and CAV2 are associated with primary open-angle glaucoma. Nat Genet. 2010; 42(10): 906-909. PMid: 20835238. https : //doi.org/10.1038/ng.661

[6] Burdon KP, Macgregor S, Hewitt AW, et al. Genome-wide association study identifies susceptibility loci for open angle glaucoma at TMCO1 and CDKN2B-AS1. Nat Genet. 2011; 43(6): 574-578. PMid: 21532571. https://doi.org/10.1038/ng.824

[7] Wei H, Wang HB. Value of optic disc formation analysis in early diagnosis of primary open angle glaucoma in basic hospital. Int $\mathrm{J}$ Ophthalmol. 2010; 10(5): 955-956.

[8] Actis AG, Belli L, Dallorto L, et al. Morphology of optic disc through heidelberg retina tomograph in retinal vein occlusions alone or in combination with primary open angle glaucoma. Open Oph- and researches aimed at different human groups lead to different results, even opposite results.

In conclusion, this study demonstrates that mutant genotypes (GA or GG) of CDKN2B (rs1063192) are associated with the increase in the susceptibility to POAG, i.e., G allele is considered to be a risk gene for POAG. The gene polymorphism of RFTN1 (rs690037), ATOH7 (rs7916697, rs3858145), CDC7 (rs1192415) and SIX (rs10483727) sites is not correlated with the susceptibility to POAG. In the subsequent studies, we will further collect cases from other regions of Inner Mongolia to obtain more information and make a layering study of the relationship between the gene polymorphism and the susceptibility to POAG in Mongolian and Han people. It will provide more clinical thoughts and reference bases for the prevention and clinical study of POAG.

\section{Acknowledgements}

We hereby express our thanks to Baogang Honspital, the First Affiliated Hospital of Baotou Medical College, Inner Mongolia International Mogolian Hospital, Baotou Eye Hospital, Baotou Chaoju Ophthalmology Hospital and Hohhot Chaoju Ophthalmology Hospital for providing the study with pathological data.

\section{Conflicts of Interest Disclosure}

The authors have no conflicts of interest related to this article.

thalmol J. 2013; 7: 34-41. PMid: 23961303. https://doi.org/ 10.2174/1874364101307010034

[9] van Koolwijk LM, Despriet DD, van Duijn CM, et al. Genetic contributions to glaucoma: heritability of intraocular pressure, retinal nerve fiber layer thickness, and optic disc morphology. Invest Ophthalmol Vis Sci. 2007; 48(8): 3669-3676. PMid: 17652737. https://doi.org/10.1167/iovs.06-1519

[10] Macgregor S, Hewitt AW, Hysi PG, et al. Genome-wide association identifies ATOH7 as a major gene determining human optic disc size. Hum Mol Genet. 2010; 19(13): 2716-2724. PMid: 20395239. https : //doi .org/10.1093/hmg/ddq144

[11] Ramdas WD, van Koolwijk LM, Ikram MK, et al. A genomewide association study of optic disc parameters. PLoS Genet. 2010; 6(6): e1000978 [2017-06-03]. Available from: http://journals.plos.org/plosgenetics/artcle?id= 10.1371/journal.pgen. 1000978

[12] Fan BJ, Wang DY, Pasquale LR, et al. Genetic variants associated with optic nerve vertical cup-to-disc ratio are risk factors for primary open angle glaucoma in a US Caucasian population. Invest Ophthalmol Vis Sci. 2011; 52(3): 1788-1792. PMid: 21398277. https://doi.org/10.1167/iovs.10-6339

[13] Cao D, Jiao X, Liu X, et al. CDKN2B polymorphism is associated with primary open-angle glaucoma (POAG) in the AfroCaribbean population of Barbados, West Indies. PLoS One. 2012; 7(6): e39278 [2017-06-09]. Available from: https ://www.ncbi. nlm.nih.gov/pubmed/22761751

[14] Shete S, Hosking FJ, Robertson LB, et al. Genome-wide association study identifies five susceptibility loci for glioma. Nat Genet. 2009; 41(8): 899-904. PMid: 19578367. https://doi.org/10.1038/ $\mathrm{ng} \cdot 407$ 
[15] Peng RB, Yu L. Research progress on TGF- $\beta$ /Smad signaling pathway in glaucoma. Rec Adv Ophthalmol. 2013; 33(12): 1190-1193.

[16] van Koolwijk LM, Despriet DD, van Duijn CM, et al. Genetic contributions to glaucoma: heritability of intraocular pressure, retinal nerve fiber layer thickness, and optic disc morphology. Invest Ophthalmol Vis Sci. 2007; 48: 3669-3676. PMid: 17652737. https:

//doi.org/10.1167/iovs.06-1519 